\title{
Host Plant-Related Parasitism and Host Feeding Activities of Diglyphus isaea (Hymenoptera: Eulophidae) on Liriomyza huidobrensis, Liriomyza sativae, and Liriomyza trifolii (Diptera: Agromyzidae)
}

\author{
Robert Musundire $^{1,2}$, Adenirin Chabi-Olaye ${ }^{1,3}$, Daisy Salifu ${ }^{1}$ and Kerstin Krüger ${ }^{2,4}$ \\ ${ }^{1}$ International Centre of Insect Physiology and Ecology (icipe), P.O. Box 30772Đ00100, GPO, Nairobi, Kenya. \\ ${ }^{2}$ Department of Zoology and Entomology, University of Pretoria, Private Bag X20, Pretoria, 0028, South \\ Africa. \\ ${ }^{3}$ Deceased January 2011. \\ ${ }^{4}$ Corresponding author, e-mail address: kkruger@zoology.up. ac.za.
}

\begin{abstract}
Host plant species can affect the behavior and attributes of parasitoids, such as host searching, oviposition, and offspring fitness. In this study, parasitism, host feeding, and sex ratios of Diglyphus isaea (Walker) (Hymenoptera: Eulophidae) on Liriomyza huidobrensis (Blanchard), Liriomyza sativae Blanchard, and Liriomyza trifolii (Burgess) (Diptera: Agromyzidae) larvae reared on Phaseolus vulgaris L., Pisum sativum L., Solanum lycopersicum L., and Vicia faba L. were determined. In no-choice tests, L. huidobrensis had the highest rate of parasitism when reared on P. vulgaris (46\%), L. sativae when reared on $V$. faba (59\%) and P. vulgaris (59\%), and L. trifolii when reared on S. lycopersicum (68\%). Host feeding in no-choice tests ranged between $2 \%$ and $36 \%$ and was highest on L. trifolii reared on $V$. faba. Results of choice tests showed a significant interaction effect for host plant and Liriomyza species on parasitism and host feeding. Within plant mixtures, L. sativae reared on $P$. vulgaris had the highest rate of parasitism (31\%), followed by L. trifolii on $S$.

lycopersicum (29\%) and L. huidobrensis on V. faba (28\%). Host feeding was highest on $L$. trifolii reared on S. lycopersicum (14\%) and lowest on L. huidobrensis reared on P. sativum and $S$. lycopersicum (1\%). In some instances, plant mixtures resulted in a higher proportion of females of $D$. isaea than single plant species. The highest proportion of females was obtained in plant mixtures on L. huidobrensis and L. trifolii on V. faba (71 and 72\%, respectively). This study suggests that planting crop mixtures can potentially lead to higher proportions of females, thus improving parasitism and host feeding, depending on Liriomyza and host plant species.
\end{abstract}

Keywords: biological control, leafminer, ectoparasitoid, sex ratio, tritrophic interactions

The leafmining flies Liriomyza huidobrensis (Blanchard), Liriomyza sativae Blanchard, and Liriomyza trifolii (Burgess) (Diptera: Agromyzidae) are economically important pests of a wide range of greenhouse and field-grown plants (Murphy and LaSalle 1999, Liu et al. 2009). These three pests are considered alien invasive species in the Afrotropical region, and $L$. trifolii was first reported in Kenya in 1976, where $10 \mathrm{yr}$ after its accidental introduction the pest was found to be widespread from the coastal regions to the highlands (Spencer 1985). The three Liriomyza species are highly polyphagous, attacking plants in several families 
(Murphy and LaSalle 1999). Globally, they are important pests of high value horticultural crops such as snow pea (Pisum sativum L.; Fabaceae), French bean (Phaseolus vulgaris L.; Fabaceae), runner bean (Phaseolus coccineus L.; Fabaceae), okra [Abelmoschus esculentus (L.) Moench; Malvaceae], aubergine (Solanum melongena L.; Solanaceae), tomato (Solanum lycopersicum L.; Solanaceae), and passion fruit (Passiflora edulis Sims; Passifloraceae), and they can cause yield losses of up to 100\% (Spencer 1973, 1990; Kotze and Dennill 1996; Chabi-Olaye et al. 2008; Gitonga et al. 2010).

Previous studies showed that natural enemies are important in regulating Liriomyza spp. populations in their native and invaded areas (Johnson 1993, Shepard et al. 1998, Murphy and LaSalle 1999, Sivapragasam et al. 1999, Thang 1999, Rauf et al. 2000, Chen et al. 2003). Diglyphus isaea (Walker) (Hymenoptera: Eulophidae) is a solitary larval ectoparasitoid of agromyzid leafminers, including L. huidobrensis, L. sativae, and L. trifolii (Ode and Heinz 2002, Liu et al. 2009). The parasitoid is used as a biological control agent against Liriomyza species in Europe, the United States, and some parts of Asia on a range of crops and ornamental plants (Ode and Heinz 2002, Liu et al. 2009). It also has been recorded in parts of Africa (Musundire et al. 2011). The highest success with $D$. isaea has been obtained with augmentative releases (Ozawa et al. 1993, 1999; Rodriguez et al. 1997). In Kenya, large-scale mass-production programs of $D$. isaea have been developed to support biological control of Liriomyza species both within the country and in South Africa.

However, the efficacy of the parasitoids depends on the ability to locate suitably sized hosts within crop habitats and to kill Liriomyza larvae through host feeding (ingestion of the contents of host larvae) or parasitism (Ode and Heinz 2002). Various attributes of host plant species can affect mate location (McAuslane et al. 1990), oviposition (Powell and Wright 1991), fecundity (Shukla and Tripathi 1993), rate of parasitism (Salvo and Valladares 2002), survival and sex ratio (Hare and Luck 1991), and body size (Salvo and Valladares 2002) of parasitoids of insect herbivores.

D. isaea and its congener Diglyphus begini (Ashmead) have been reported to host feed, lay eggs on hosts (parasitize), and allocate sex based on the quality of larval hosts (Heinz and Parrella 1989, Ode and Heinz 2002). Therefore, host feeding behavior, parasitism, and sex allocation of parasitoids can indirectly be influenced by the host plant, Liriomyza species, and the larval size of the host (Johnson and Hara 1987; Ode and Heinz 2002, Salvo and Valladares 2002).

Few studies focused on the interaction between the three polyphagous Liriomyza species and $D$. isaea in agroecosytems. The current study determined parasitism, host feeding, and proportion of female progeny of $D$. isaea on three Liriomyza species reared on P. vulgaris, $P$. sativum, S. lycopersicum, and Vicia faba L. (Fabaceae) We evaluated the implications of the findings of the current study for biological control of Liriomyza species with $D$. isaea and for effective mass rearing of the parasitoid.

\section{Materials and Methods}

\section{Plants.}

Four plant species- $P$. vulgaris ('Julia'), $P$. sativum ('Oregon Sugar Pod III'), $S$. lycopersicum ('Moneymaker'), and $V$. faba (a local Kenyan open-pollinated cultivar) -were used in the experiments. Plants were grown in red clay potting soil in plastic pots $(11 \mathrm{~cm}$ in 
diameter by $9 \mathrm{~cm}$ in depth) and maintained in a Liriomyza-free screenhouse at $27 \pm 2^{\circ} \mathrm{C}$ and $\approx 30 \% \mathrm{RH}$ at icipe in Nairobi, Kenya. One gram of a top-dressing of calcium ammonium nitrate ( $27 \%$ nitrogen) was added per pot $1 \mathrm{wk}$ after germination or transplanting of plants.

Larval density on the four host plant species is strongly positively related to leaf area (Musundire et al. 2012). However, larval densities did not vary significantly among the plant species tested when the leaf area ranged between 50 and $70 \mathrm{~cm}^{2}$ (Musundire et al. 2012). Thus, the plant species used were standardized by using the same age, size, fertilization regime, and leaf area (50-70 $\left.\mathrm{cm}^{2}\right)$. Two-week-old $P$. vulgaris, $P$. sativum, and $V$. faba plants and 5-wk-old $S$. lycopersicum plants were used in experiments.

\section{Insect Rearing.}

L. huidobrensis, L. trifolii, and L. sativae were obtained from cultures maintained at the insectary of icipe. The three species were reared at $27 \pm 0.6^{\circ} \mathrm{C}, 27-35 \% \mathrm{RH}$, and a photoperiod 12:12 (L:D) h. L. huidobrensis was reared on V. faba, and $L$. sativae and $L$. trifolii were reared on $P$. vulgaris. All species had been reared on the respective plant species for $\approx 18-20$ generations before the experiments. To avoid bias regarding the original host plant species on which the insects were reared, each of the three Liriomyza species from the cultures was further reared on each of the four host plants for three generations before use in experiments. L. sativae and L. trifolii did not produce enough progeny on $P$. sativum; hence, the effect of this host plant species on these two species was not evaluated in some cases.

For each treatment, 16 potted plants of each of the four plant species were infested with $504-$ d-old adult male and female Liriomyza (sex ratio, 1:1). Liriomyza adults together with the respective potted plant species were kept in ventilated cages ( 50 by 50 by $45 \mathrm{~cm}$ ) and fed on a $10 \%$ sucrose solution while given an oviposition period of $4 \mathrm{~h}$. This exposure method allowed the development of almost the same aged cohort of larvae. Thereafter, plants were removed and transferred to another similar cage free of adult Liriomyza leafminers to monitor the development of larvae until the late second or third instar (8-10 d, depending on host plant species). Infested potted plants with late second- and third-instar larvae were used for the experiments.

D. isaea used in the experiments were supplied by Dudutech (K) Pvt (Ltd), Naivasha, Kenya, and the identification was confirmed by C. D. Zhu (Institute of Zoology, Chinese Academy of Sciences, Beijing, China). These parasitoids were mass reared on L. huidobrensis on $P$. sativum under uniform greenhouse conditions. Parasitoids were allowed to mate for a period of $48 \mathrm{~h}$ in ventilated cages ( 40 by 20 by $20 \mathrm{~cm}$ ) and then given a preoviposition period of 12 $\mathrm{h}$ and supplied with a $10 \%$ honey solution throughout mating and preoviposition. Parasitoids from a single batch were used in the experiments.

\section{Effect of Host Plant on Parasitism and Host Feeding of $D$. isaea on Larvae of Liriomyza Species.}

No-Choice Test. Four potted plants of the same plant species infested with the same Liriomyza species were placed together in a ventilated Perspex cage (50 by 50 by $45 \mathrm{~cm}$ ). Forty-five randomly selected premated $D$. isaea females were released per cage for $24 \mathrm{~h}$ as opposed to single parasitoids to simulate the releases for mass rearing and field applications. After the 24-h period, plants were transferred to similar-sized cages free of parasitoids to allow for the development of $D$. isaea from parasitized Liriomyza larvae. The experiment was 
replicated four times for each host plant and Liriomyza species combination using individuals reared from each host and using these for testing on the respective plant species.

Choice Test. Four potted plants consisting of one of each of the four plant species $P$. vulgaris, $P$. sativum, $S$. lycopersicum, and $V$. faba, each infested with live late second- to third-instar larvae of the same Liriomyza species, were placed in ventilated Perspex cages ( 50 by 50 by $45 \mathrm{~cm}$ ). As in the no-choice test, 45 premated $D$. isaea were released per cage for $24 \mathrm{~h}$. The experiment was replicated four times for each host plant and Liriomyza species combination using individuals reared from each host and using these for testing on the respective plant species.

The no-choice and choice tests were carried out in the laboratory under the same environmental conditions as described under Insect Rearing. Four days after exposure to $D$. isaea, all mines on leaves were dissected under an EZ4D stereomicroscope (Leica Microsystems, GmbH, Wetzlar, Germany). The total numbers of parasitized and host-fed larvae and of unparasitized larvae were determined for each plant and Liriomyza species combination.

Larvae were recorded as host fed once they became flaccid with black spots on their body as a result of stings of parasitoid females and parasitized when they were found with immatures of $D$. isaea (Minkenberg 1989). D. isaea is a destructive nonconcurrent host feeder (Ode and Heinz 2002). Therefore, the methods used in this study were adequate to assess host feeding effects and parasitism as separate aspects.

\section{Effect of Host Plant on Sex Ratio of $D$. isaea.}

To determine the sex ratio of $D$. isaea on different host plants and Liriomyza species, infested plants were kept for $7 \mathrm{~d}$ after exposure to parasitoids to allow for complete development of both unparasitized Liriomyza larvae and parasitoids from parasitized larvae. After $7 \mathrm{~d}$, infested leaves were harvested and incubated in ventilated cages ( 40 by 20 by $20 \mathrm{~cm}$ ) under the same laboratory conditions as in the previous experiments until the emergence of adult $D$. isaea or Liriomyza species.

Three days after the first adult emergence of $D$. isaea (the period during which all adults from the incubations were expected to have emerged), all adults were killed by instant freezing. They were sexed using the key developed by Bouček (1988). Adults of $D$. isaea display a sexually dimorphic color pattern of the hind tibia. The proportions of male and female progeny were determined for all plant and Liriomyza species in the choice and the no-choice tests. However, due to difficulties in rearing $L$. sativae and $L$. trifolii on $P$. sativum, very few larvae were available for parasitism; hence, very few or no $D$. isaea progeny were recovered from this host plant species. Because of the small sample sizes, $P$. sativum was excluded from some analyses.

\section{Data Analyses.}

All data were analyzed with SAS (SAS Institute 2002-2003). To test for density dependence between the initial number of larvae and parasitism, Pearson correlation coefficients, by using PROC CORR, were computed between the number of Liriomyza larvae parasitized and the total number of Liriomyza larvae per replicate and percentage of parasitism in choice and no- 
choice experiments. Host feeding levels were consistently low in both experiments; therefore, the relationship between host feeding and leafminer density could not be determined.

In the no-choice test, a logistic regression model was used to examine the effect of host plant cultivars, Liriomyza species, and their interaction on the number of larvae that were either parasitized or host fed out of the total number of larvae observed per treatment, by using PROC GENMOD (SAS Institute 2002-2003). Mean percentages of parasitized and host-fed larvae were separated using a pairwise chi-square test (Cox and Key 1993). In the choice test, the number of parasitized larvae and the number of host-fed larvae per host plant species were analyzed using a multinomial logistic regression model with the link function GLOGIT where $V$. faba was used as a reference outcome category by using PROC LOGISTIC. The sex ratio of $D$. isaea was determined as proportion of females out of the total number of parasitoids (males and females), and a logistic regression model was used to evaluate the effect of host plants, Liriomyza species, and their interaction on the sex ratios. Mean percentage of sex ratios were separated using a pairwise chi-square test (Cox and Key 1993).

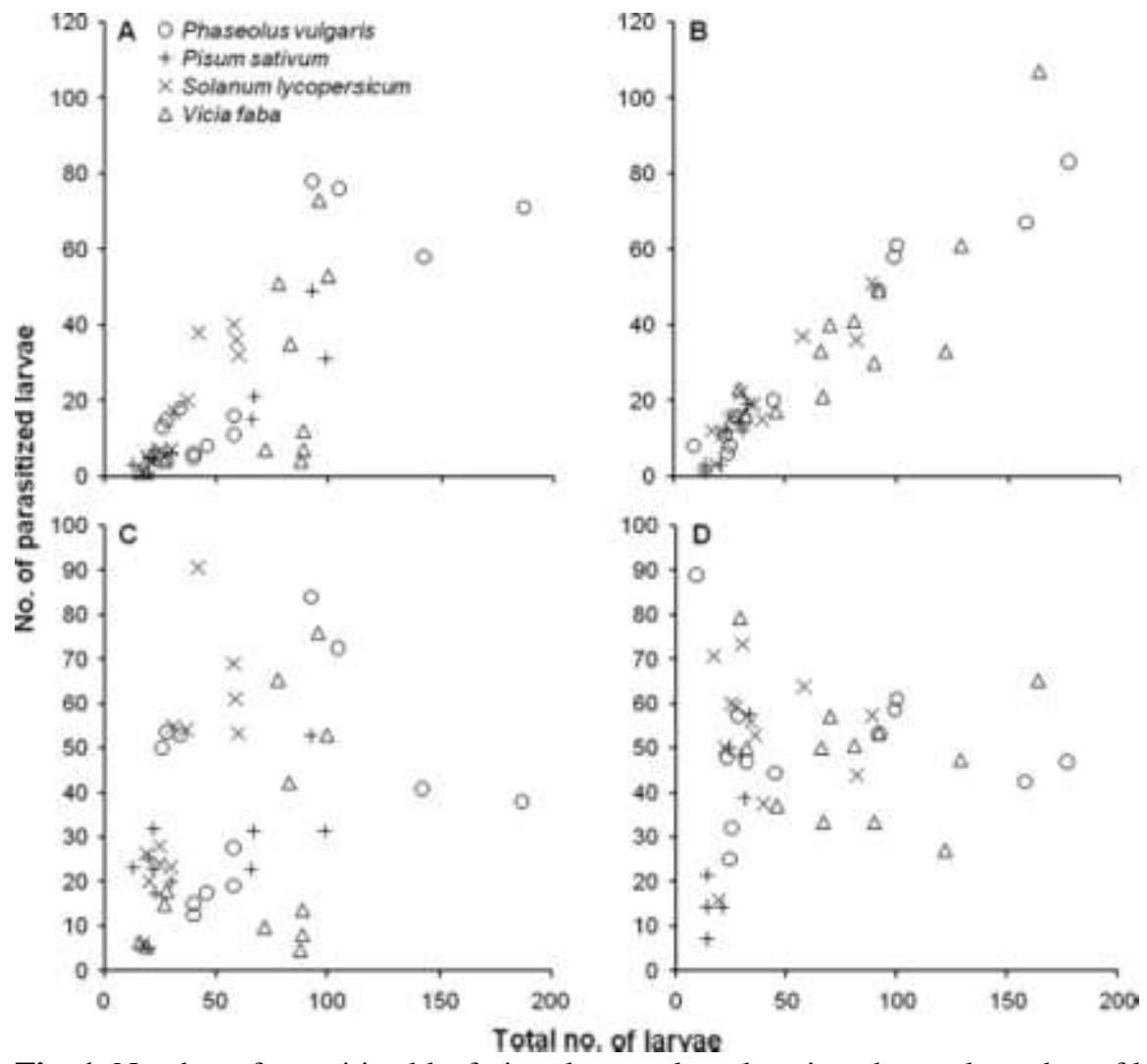

Fig. 1. Number of parasitized leafminer larvae plotted against the total number of larvae per replicate in choice (A) and no-choice (B) experiments and percentage of larvae parasitized plotted against the total number larvae per replicate in choice $(\mathrm{C})$ and no-choice $(\mathrm{D})$ experiments. 


\section{Results}

\section{Effect of Total Number of Larvae per Replicate on Total Number and Proportion of Parasitized Larvae.}

There was a strong positive linear relationship between the total number of parasitized larvae and total number of larvae available for host feeding and parasitism in the choice $(r=0.95)$ and no-choice $(r=0.77)$ experiments (Fig. 1A and B). However, there was no such trend for the percentage of larvae parasitized and the total number of larvae for either choice $(r=0.33)$ or no choice experiment $(r=0.29$ ) (Fig. 1C and D), suggesting that the number of available larvae did not influence percentage parasitism.

\section{Effect of Host Plant on D. isaea Parasitism and Host Feeding on Larvae of Liriomyza.}

No-Choice Test. There is strong evidence for Liriomyza and host plant species interaction on the rate of parasitism by $D$. isaea $\left(\chi^{2}=193.88, \mathrm{df}=6, P<0.0001\right)$. The highest rate of parasitism of L. huidobrensis was recorded on P. vulgaris, with a mean of $46 \%$, followed by P. sativum (23\%), S. lycopersicum (20\%), and V. faba (9\%) (Table 1). D. isaea parasitized a significantly higher number of $L$. sativae larvae on $V$. faba (59\%) and P. vulgaris (59\%), compared with those reared on P. sativum (35\%) and S. lycopersicum (39\%). The rate of parasitism of $L$. trifolii was significantly higher on $S$. lycopersicum $(68 \%)$ compared with $P$. vulgaris (16\%), P. sativum (19\%), and $V$. faba (11\%) (Table 1). The percentage of parasitism by $D$. isaea was higher on $L$. sativae compared with $L$. huidobrensis and L. trifolii on all plant species except for $S$. lycopersicum, where it was highest for L. trifolii (Table 1).

The percentage of larvae host fed by $D$. isaea was generally low across all the host plant cultivars and Liriomyza species compared with that of parasitism. It was 0.3-3.2 times lower on V. faba and P. vulgaris, 1.8-3.6 times lower on S. lycopersicum, and 4-10 times lower on $P$. sativum. Logistic regression analysis showed a highly significant interaction effect between host plant cultivars and Liriomyza species $\left(\chi^{2}=25.44, \mathrm{df}=6, P=0.0003\right)$. The highest host feeding level was recorded on L. trifolii reared on V. faba $(36 \%)$ and P. vulgaris (34\%) and lowest on all Liriomyza species reared on P. sativum (2-5\%) (Table 1). Host feeding activity by $D$. isaea on $L$. huidobrensis was higher on $P$. vulgaris and S. lycopersicum compared with $P$. sativum and $V$. faba. However, $L$. sativae larvae reared on $P$. vulgaris, $S$. lycopersicum, and $V$. faba were equally host fed. The level of host feeding on L. trifolii was significantly higher on $P$. vulgaris and $V$. faba compared with $P$. sativum and $S$. lycopersicum (Table 1).

Choice Test. The choice of host plant species by $D$. isaea varied significantly between Liriomyza species (parasitism: Wald $\chi^{2}=297.3, \mathrm{df}=6, P<0.0001$; host feeding: Wald $\chi^{2}=$ 129.2, df $=6, P<0.0001)$. Parasitism was highest on L. sativae reared on $P$. vulgaris $(31 \%)$, L. trifolii reared on S. lycopersicum (29\%), and L. huidobrensis reared on V.faba (28\%) (Fig. 2A). Host feeding in general was highest on L. trifolii reared on S. lycopersicum (14\%) and lowest on L. huidobrensis (1\%) reared on P. sativum and S. lycopersicum (Fig. 2B). 
Table 1. Percentage parasitism and host feeding (mean \pm SEM) by D. isaea on three Liriomyza leafminer species reared on four host plant species in a no-choice test

\begin{tabular}{|c|c|c|c|c|}
\hline $\begin{array}{c}\text { Liriomyza } \\
\text { species }\end{array}$ & P. sativum & P. vulgaris & S. lycopersicum & V. faba \\
\hline & \multicolumn{4}{|c|}{$\%$ parasitism of Liriomyza larvae by $D$. isaea } \\
\hline L. huidobrensis & $22.9 \pm 1.6 \mathrm{bB}$ & $46.0 \pm 6.2 \mathrm{aA}$ & $20.4 \pm 1.3 \mathrm{bC}$ & $8.9 \pm 0.6 \mathrm{cB}$ \\
\hline L. sativae & $34.7 \pm 2.8 \mathrm{bA}$ & $58.9 \pm 4.5 \mathrm{aA}$ & $39.2 \pm 2.5 \mathrm{bB}$ & $59.4 \pm 4.9 \mathrm{aA}$ \\
\hline \multirow[t]{2}{*}{ L. trifolii } & $19.0 \pm 4.7 \mathrm{bB}$ & $16.0 \pm 1.4 \mathrm{bB}$ & $68.4 \pm 8.0 \mathrm{aA}$ & $11.0 \pm 3.1 \mathrm{bB}$ \\
\hline & \multicolumn{4}{|c|}{ \% Liriomyza larvae host fed by $D$. isaea } \\
\hline L. huidobrensis & $2.2 \pm 1.3 \mathrm{bA}$ & $14.8 \pm 1.5 \mathrm{aB}$ & $11.4 \pm 0.5 \mathrm{aB}$ & $4.7 \pm 1.5 \mathrm{bC}$ \\
\hline L. sativae & $5.2 \pm 1.9 \mathrm{bA}$ & $18.6 \pm 3.2 \mathrm{aB}$ & $19.7 \pm 1.2 \mathrm{aA}$ & $18.4 \pm 2.0 \mathrm{aB}$ \\
\hline L. trifolii & $5.1 \pm 3.6 \mathrm{cA}$ & $33.6 \pm 2.1 \mathrm{aA}$ & $19.0 \pm 3.3 \mathrm{bA}$ & $36.4 \pm 4.1 \mathrm{aA}$ \\
\hline
\end{tabular}



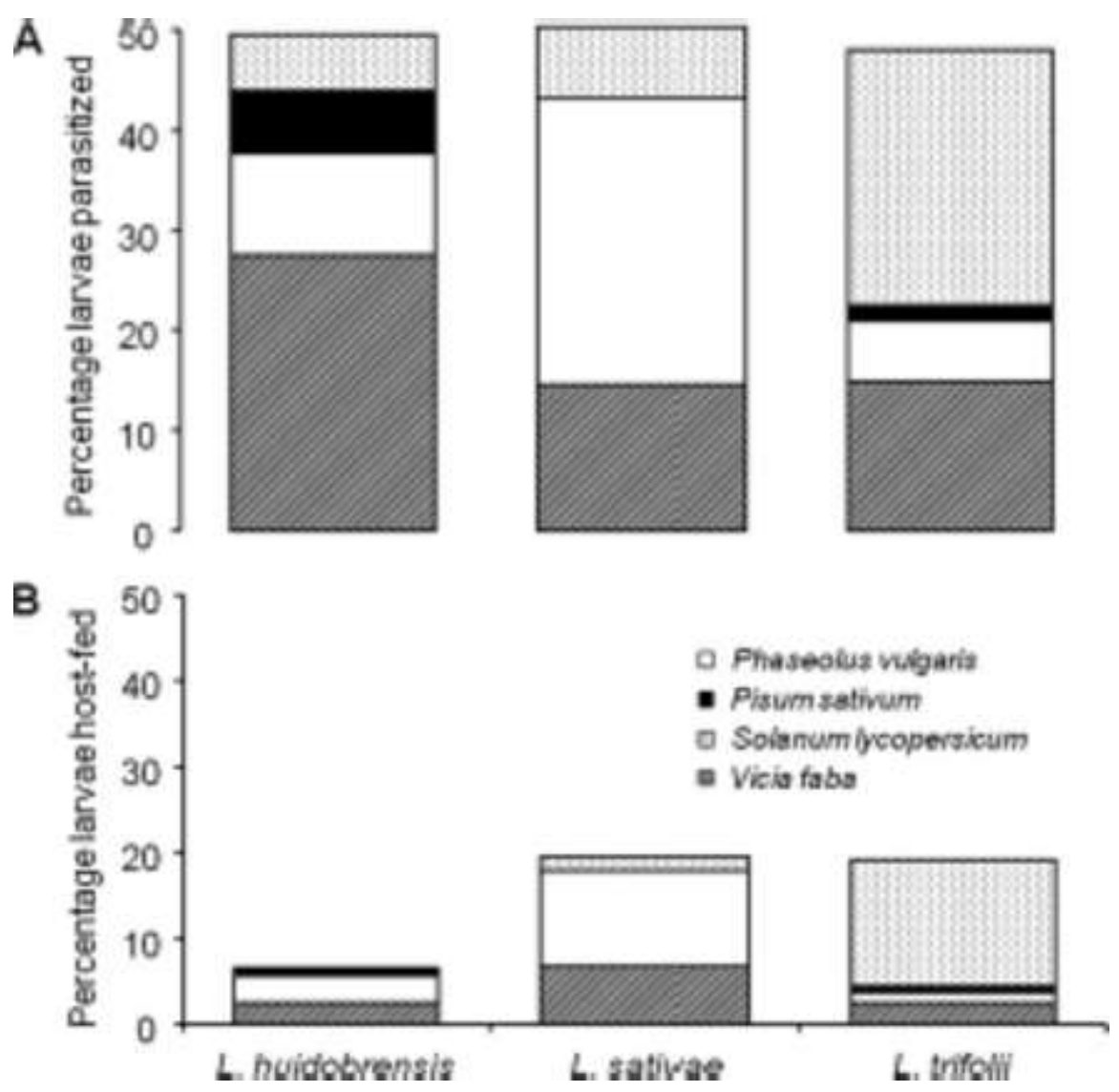

Fig. 2. Percentage of Liriomyza larvae parasitized (A) and host fed (B) by D. isaea in choice experiments.

\section{Sex Ratio of $D$. isaea.}

In the no-choice test, sex ratios of $D$. isaea varied significantly with host plant $\left(\chi^{2}=22.26\right.$, df $=3, P<0.001)$ and Liriomyza species $\left(\chi^{2}=14.1, \mathrm{df}=2, P<0.001\right)$. No or few D. isaea developed into adults from $L$. sativae and $L$. trifolii reared on P. sativum. Across Liriomyza species, the highest proportion of females was recorded on L. huidobrensis reared on $P$. sativum (64\%) and lowest on L. sativae reared on S. lycopersicum (25\%) (Table 2).

In the choice test, there was a significant interaction effect between host plant cultivars and Liriomyza species on sex ratios $\left(\chi^{2}=47.53, \mathrm{df}=4, P=0.001\right)$. D. isaea yielded a higher percentage of female progeny from L. huidobrensis and L. trifolii reared on V. faba (71 and $72 \%$, respectively), whereas the highest proportion of female $D$. isaea from $L$. sativae was obtained from larvae reared on S. lycopersicum (50\%).

A comparison of the sex ratios between the no-choice and choice experiments showed that the percentage of female progeny of $D$. isaea from $L$. huidobrensis reared on $V$. faba increased from $52 \%$ in the no-choice test to $71 \%$ in the choice test (Table 2). An increase also was observed for the female-biased sex ratio of $D$. isaea from $L$. sativae reared on $S$. lycopersicum, which increased from $25 \%$ in the no-choice test to $50 \%$ in the choice test (Table 2). For L. trifolii reared on $V$. faba, there was also an increase in the female-biased sex ratio of $D$. isaea from $53 \%$ in the no-choice test to $72 \%$ in the choice test (Table 2). 
Table 2. Percentage of female offspring of $D$. isaea in choice and no-choice tests

\begin{tabular}{|c|c|c|c|c|c|c|}
\hline \multirow{2}{*}{ Host plant } & \multicolumn{2}{|c|}{ L. huidobrensis } & \multicolumn{2}{|c|}{ L. sativae } & \multicolumn{2}{|c|}{ L. trifolii } \\
\hline & Choice & No-choice & Choice & No-choice & Choice & No-choice \\
\hline P. sativum & $52.7 \pm 5.7 \mathrm{aB}$ & $63.5 \pm 4.7 \mathrm{aA}$ & & & & \\
\hline P. vulgaris & $48.3 \pm 3.5 \mathrm{aBC}$ & $53.2 \pm 5.4 \mathrm{aA}$ & $35.5 \pm 6.3 \mathrm{bA}$ & $53.4 \pm 4.3 \mathrm{aA}$ & $34.2 \pm 3.8 \mathrm{bB}$ & $52.0 \pm 5.2 \mathrm{aA}$ \\
\hline S. lycopersicum & $36.3 \pm 1.5 \mathrm{bC}$ & $46.0 \pm 3.8 \mathrm{aB}$ & $49.7 \pm 2.5 \mathrm{aA}$ & $25.4 \pm 2.3 \mathrm{bC}$ & $37.0 \pm 1.9 \mathrm{bB}$ & $30.4 \pm 4.4 \mathrm{bB}$ \\
\hline V. faba & $71.3 \pm 3.7 \mathrm{aA}$ & $52.0 \pm 2.4 \mathrm{bA}$ & $30.6 \pm 2.9 \mathrm{bB}$ & $37.4 \pm 2.1 \mathrm{aB}$ & $72.2 \pm 3.2 \mathrm{aA}$ & $53.4 \pm 1.2 \mathrm{bA}$ \\
\hline
\end{tabular}




\section{Discussion}

Females of $D$. isaea and $D$. begini, a close relative of $D$. isaea, oviposit on larger hosts but reject or host feed on smaller hosts (Heinz and Parrella 1989, Ode and Heinz 2002). Results of the current study suggest that the rate of parasitism may not necessarily depend on host size alone but also on other host plant characteristics. This is supported by the observation that L. huidobrensis, the largest of the three Liriomyza species (Spencer 1973, Musundire et al. 2012), did not have the highest rate of parasitism in the no-choice and choice experiments in the current study. In addition, using the same host plant species and cultivars as in the current study, V. faba seemed a more suitable host plant for L. trifolii than P. vulgaris or $S$. lycopersicum based on adult size as a measure of performance (Musundire et al. 2012), whereas parasitism was highest on S. lycopersicum. The disparity between host plant-related size variation in adult Liriomyza species (Musundire et al. 2012) and the current results for leafminer-plant-related variation in parasitism and host feeding levels could be attributed to several factors, including volatile cues released by leafminer-damaged host plants (FinidoriLogli et al. 1996, Zhao and Kang 2002, Wei et al. 2006) and visual cues, such as the shape of a leaf mine (Sugimoto et al. 1988).

Agromyzid leafmines can take different shapes depending on the species concerned (Spencer and Steyskal 1986). Salvo and Valladares (2004), who examined the role of mine shape and color contrast on the parasitoid assemblages of agromyzid leafminers in Central Argentina, observed that mine shape affected parasitism rates by specialist and generalist parasitoids. In the current study, there was considerable inter- and intraspecific variation in mine shape and appearance of different host plants attacked by different Liriomyza species (R. M., personal observation). These differences could have been a potential cause for host plant-related variation in parasitism and host feeding as mine shape and appearance have been reported to play an important role in aiding host finding of parasitoids (Sugimoto et al. 1988).

Differential host plant use and differential effects of natural enemies have been suggested as contributing factors to displacement among Liriomyza species in the United States (Reitz and Trumble 2002a,b) and Japan (Abe and Tokumaru 2008). The results of the current study partially explain the roles that can be played by both host plants and parasitoids in the displacement of Liriomyza species. For example, Musundire et al. (2012) did not observe size differences in L. huidobrensis when reared on the same host plant species and cultivars used in the current study. However, parasitism and host feeding by $D$. isaea was highest on $L$. huidobrensis when reared on $P$. vulgaris, suggesting differential host plant-based preferences by the parasitoid.

In general, studies have shown that host feeding can contribute considerably to total parasitoid-inflicted mortality (Legner 1979, van Driesche and Taub 1983, Kidd and Jervis 1989). In the current study, host feeding levels were relatively low (2-36\%) compared with results of other studies; the highest host feeding rate (36\%) was recorded for L. trifolii on $V$. faba. In contrast to the current study, a higher number of $L$. trifolii larvae were host fed than parasitized by both introduced and native $D$. isaea (Hondo et al. 2006). The differences between this and other studies could be due to differences in larval densities, and consequently larval host size, because smaller larvae tend to be host fed rather than parasitized (Ode and Heinz 2002).

The relatively high level of host feeding of $L$. trifolii on $S$. lycopersicum in the choice test compared with the same species on $V$. faba was matched by a higher rate of parasitism of the 
same Liriomyza species on $S$. lycopersicum compared with $V$. faba. The exact mechanism involved requires further investigation.

However, despite the relatively low levels of host feeding of $D$. isaea across the host plants and Liriomyza species combinations evaluated, host feeding still has important consequences for biological control for both inoculative releases and mass rearing of $D$. isaea.

Theoretically, reduced levels of host feeding imply a better chance of parasitism, directly leading to an increase in parasitoid numbers as opposed to high host feeding levels that do not result in a population build-up (Ode and Heinz 2002). However, despite apparent low levels of host feeding observed in the current study, from a pest management perspective, host feeding has an additive effect on the total mortality imposed by $D$. isaea.

Host quality, host feeding, and nonhost diet can influence sex allocation by female solitary parasitoid wasps (Charnov 1982, King 1987, Kidd and Jervis 1989, Godfray 1994, Ode and Hardy 2008). A carbohydrate-rich diet, e.g., honey, may increase the proportion of female progeny of parasitoids (Onagbola et al. 2007). In this study, parasitoids were provided with a $10 \%$ honey solution during the preoviposition and mating periods. Thus, the parasitoids were provided with sufficient food resources to avoid a sex bias toward males due to factors other than those associated with the Liriomyza leafminer host quality and host plant-related factors.

The proportion of females in any $D$. isaea population in augmentative field releases and mass rearing is important as females are more valuable than males because they are directly responsible for killing pests by oviposition, host feeding, or both (Ode and Heinz 2002). The highest proportion of females of $D$. isaea across all the host plant species was observed for $L$. huidobrensis. Although host sizes of Liriomyza leafminer larvae were not measured in this study, studies by Spencer (1973) and Musundire et al. (2012) showed that under uniform conditions, L. huidobrensis larvae are larger than those of $L$. sativae and $L$. trifolii, suggesting that this species should receive a higher allocation of female progeny compared with larvae of $L$. sativae and L. trifolii. In the current study, the proportion of females depended on host plant and Liriomyza species tested and varied between 25\% (L. sativae on S. lycopersicum) and $72 \%$ (L. trifolii on V. faba). Except for D. isaea populations recovered from L. trifolii reared on S. lycopersicum and L. sativae on $V$. faba, the proportion of females (46-72\%) was higher or comparable with research results obtained in other studies on sex ratios for massreared parasitoids, e.g., 45\% (Ode and Heinz 2002), 56\% (Chow and Heinz 2005), and 47\% (Parrella et al. 1989). The results of the current study on the relationships among host plant, Liriomyza species, and $D$. isaea can be used for further larger scale field studies aimed at optimizing field releases and adapting mass rearing of $D$. isaea on these plant species.

In conclusion, parasitism and host feeding levels by $D$. isaea obtained in this study depended on Liriomyza and host plant combination. L. trifolii reared on $V$. faba plants were preferred for host feeding in no-choice experiments, whereas L. trifolii on S. lycopersicum were the preferred host for parasitism in no-choice experiments. Plant mixtures in some instances altered the parasitism, host feeding levels and sex ratio of $D$. isaea. There is therefore a greater scope for evaluating the role of intercropping in the manipulation of parasitism, host feeding, and sex ratios of $D$. isaea for biological control of Liriomyza species in greenhouses and open fields. 


\section{Acknowledgments}

We thank S. A. Mohamed (icipe) for critically reviewing the manuscript and anonymous referees for constructive comments. This work was supported by icipe's research project on integrated pest management of vegetable leafminers through the African Regional Postgraduate Programme in Insect Science (ARPPIS), the German Academic Exchange Programme (DAAD), icipe, and the University of Pretoria. The leafminer management project was funded by the German Federal Ministry for Economic Cooperation and Development (BMZ).

\section{References Cited}

Abe Y., Tokumaru S. 2008. Displacement in two invasive species of leafminer fly in different localities. Biol. Invasions 10: 951-953.

Bouček Z. 1988. Australasian Chalcidoidea (Hymenoptera). A biosystematic revision of genera of fourteen families, with a reclassification of species. CAB International, Wallingford, Oxon, United Kingdom.

Chabi-Olaye A., Mujica N., Löhr B., Kroschel J. 2008. Role of agroecosystem in the abundance and diversity of Liriomyza leafmining flies and their natural enemies. In Abstracts of the XXIII International Congress of Entomology, 6-12 July 2008, Durban, South Africa.

Charnov E. L. 1982. The theory of sex allocation. Princeton University Press, Princeton, NJ.

Chen X.-X., Lang F.-Y., XU Z.-H., He J.-H., Ma Y. 2003. The occurrence of leafminers and their parasitoids on vegetables and weeds in Hangzhou area, Southeast China. Biocontrol 48: 515-527.

Chow A., Heinz K. M. 2005. Using hosts of mixed sizes to reduce male-biased sex ratio in the parasitoid wasp, Diglyphus isaea. Entomol. Exp. Appl. 117: 193-199.

Cox M. K., Key C. H. 1993. Post hoc pair-wise comparisons for the chi-square test of homogeneity of proportions. Educ. Psychol. Meas. 53: 951-962.

Finidori-Logli V., Bagnéres A.-G., Clément J.-L. 1996. Role of plant volatiles in the search for a host by parasitoid Diglyphus isaea (Hymenoptera: Eulophidae). J. Chem. Ecol. 22: 541558.

Gitonga Z. M., Chabi-Olaye A., Mithöfer D., Okello J. J., Ritho C. N. 2010. Control of invasive Liriomyza leafminer species and compliance with food safety standards by small scale snow pea farmers in Kenya. Crop Prot. 29: 1472-1477.

Godfray H.C.J. 1994. Parasitoids: behavioral and evolutionary ecology. Princeton University Press, Princeton, NJ.

Hare J. D., Luck R. F.. 1991. Indirect effects of citrus cultivars on life history parameters of a parasitic wasp. Ecology 72: 1576-1585. 
Heinz K. M., Parrella M. P. 1989. Attack behavior and host size selection by Diglyphus begini on Liriomyza trifolii in chrysanthemum. Entomol. Exp. Appl. 53: 147-156.

Hondo T., Koike A., Sugimoto T. 2006. Comparison of thermal tolerance of seven native species of parasitoids (Hymenoptera: Eulophidae) as biological control agents against Liriomyza trifolii (Diptera: Agromyzidae) in Japan. Appl. Entomol. Zool. 41: 73-82.

Johnson M. W. 1993. Biological control of Liriomyza leafminers in the Pacific Basin. Micronesica Suppl. 4: 81-92.

Johnson M. W., Hara A. H. 1987. Influence of host crop on parasitoids (Hymenoptera) of Liriomyza spp. (Diptera: Agromyzidae). Environ. Entomol. 16: 339-344.

Kidd N.A.C., Jervis M. A. 1989. The effects of host-feeding behaviour on the dynamics of parasitoid-host interactions, and the implications for biological control. Res. Popul. Ecol. 31: 235-274.

King B. H.. 1987. Offspring sex ratios in parasitoid wasps. Q. Rev. Biol. 62: 367-396.

Kotze D. J., Dennill G. B. 1996. The effect of Liriomyza trifolii (Burgess) (Dipt., Agromyzidae) on fruit production and growth of tomatoes, Lycopersicon esculentum (Mill). (Solanaceae). J. Appl. Entomol. 120: 231-235.

Legner E. F. 1979. The relationship between host destruction and parasite reproductive potential in Muscidifurax raptor, M. zaraptor, and Spalangia endius (Chalcidoidea: Pteromalidae). Entomophaga 24: 145-152.

Liu T.-X., Kang L., Heinz K. M., Trumble J. 2009. Biological control of Liriomyza leafminers: progress and perspective. CAB Rev. Persecpt. Agric. Vet. Sci. Nutr. Nat. Resour. 4: $1-16$.

McAuslane H. J., Vinson S. B., Williams H. J.. 1990. Influence of host plant on mate location by the parasitoid Campoletis sonorensis (Hymenoptera: Ichneumonidae). Environ. Entomol. 19: 26-31.

Minkenberg O.P.J.M. 1989. Temperature effects on the life history of the eulophid wasp Diglyphus isaea, an ectoparasitoid of leafminers (Liriomyza spp.), on tomatoes. Ann. Appl. Biol. 115: 381-397.

Murphy S. T., LaSalle J. 1999. Balancing biological control strategies in the IPM of New World invasive Liriomyza leafminers in field vegetable crops. Biocontrol News Inf. 20: 91104.

Musundire R., Chabi-Olaye A., Krüger K.. 2012. Host plant effects on morphometric characteristics of Liriomyza huidobrensis, L. sativae and L. trifolii (Diptera: Agromyzidae). J. Appl. Entomol. (DOI 10.1111/j.1439-0418.2010.01597.x).

Musundire R., Chabi-Olaye A., Löhr B., Krüger K. 2011. Diversity of Agromyzidae and associated hymenopteran parasitoid species in the Afrotropical region: implications for biological control. Biocontrol 56: 1-9. 
Ode P. J., Hardy I.C.W. 2008. Parasitoid sex ratios and biological control, pp. 253-291. In Wajnberg É., Bernstein C., van Alphen J. (eds.), Behavioral ecology of insect parasitoids: from theoretical approaches to field applications. Blackwell Publishing, Oxford, United Kingdom.

Ode P. J., Heinz K. M.. 2002. Host-size-dependent sex ratio theory and improving massreared parasitoid sex ratios. Biol. Control 24: 31-41.

Onagbola E. O., Fadamiro H. Y., Mbata G. N. 2007. Longevity, fecundity, and progeny sex ratio of Pteromalus cerealellae in relation to diet, host provision, and mating. Biol. Control 40: $222-229$.

Ozawa A., Kobayashi H., Amano T., Ikari T., Saito T. 1993. Evaluation of imported parasitic wasps as biological control agents of the legume leafminer, Liriomyza trifolii Burgess, in Japan. II. A field test on cherry tomatoes in a plastic greenhouse, in Shizuoka Prefecture. Proc. Kanto-Tosan Plant Prot. Soc. 40: 239-241.

Ozawa A., Saito T., Ota M. 1999. Biological control of the American serpentine leafminer, Liriomyza trifolii (Burgess), on tomato in greenhouses by parasitoids. I. Evaluation of biological control by release of Diglyphus isaea (Walker) in experimental greenhouses. Jpn. J. Appl. Entomol. Zool. 43: 161-168.

Parrella M. P., Yost J. T., Heinz K. M., Ferrentino G. W. 1989. Mass rearing of Diglyphus begini (Hymenoptera: Eulophidae) for biological control of Liriomyza trifolii (Diptera: Agromyzidae). J. Econ. Entomol. 82: 420-425.

Powell W., Wright A. F. 1991. The influence of host food plants on host recognition by four aphiidine parasitoids (Hymenoptera: Braconidae). Bull. Entomol. Res. 81: 449-453.

Rauf A., Shepard B. M., Johnson M. W. 2000. Leafminers in vegetables, ornamental plants and weeds in Indonesia: surveys of host crops, species composition and parasitoids. Int. J. Pest Manag. 46: 257-266.

Reitz S. R., Trumble J. T. 2002a. Competitive displacement among insects and arachnids. Annu. Rev. Entomol. 47: 435-465.

Reitz S. R., Trumble J. T. 2002b. Interspecific and intraspecific differences in two Liriomyza leafminer species in California. Entomol. Exp. Appl. 102: 101-113.

Rodriguez J. M., Rodriguez R., Florido A., Hernandez R. 1997. Integrated pest management on tomatoes in Gran Canaria (Canary Islands). IOBC/WPRS Bull. 20: 39-44.

Salvo A., Valladares G. 2002. Plant-related intraspecific size variation in parasitoids (Hymenoptera: Parasitica) of a polyphagous leafminer (Diptera: Agromyzidae). Environ. Entomol. 31: 874-879.

Salvo A., Valladares G. R. 2004. Looks are important: parasitic assemblages of agromyzid leafminers (Diptera) in relation to mine shape and contrast. J. Anim. Ecol. 73: 494-505.

SAS Institute. 2002-2003. SAS version 9.1.3. SAS Institute, Cary, NC. 
Shepard B., Samsudin M., Braun A. R.. 1998. Seasonal incidence of Liriomyza huidobrensis (Diptera: Agromyzidae) and its parasitoids on vegetables in Indonesia. Int. J. Pest Manag. 44: 43-47.

Shukla A. N., Tripathi C.P.M. 1993. Effect of food plant on the offspring sex ratio of Diaretiella rapae (Hymenoptera: Aphididae), a parasitoid of Lipaphis erysimi Kalt (Hemiptera: Aphididae). Biol. Agric. Hortic. 9: 137-146.

Sivapragasam A., Syed A. R., LaSalle J., Ruwaida M. 1999. Parasitoids of invasive agromyzid leafminers on vegetables in Peninsular Malaysia, pp. 127-128. In Proceedings of the Symposium on Biological Control in the Tropics, MARDI Training Center, 18-19 March 1999, Serdang, Selangor, Malaysia.

Spencer K. A. 1973. Agromyzidae (Diptera) of economic importance. In Series Entomologica 9. Dr. W. Junk, The Hague, Netherlands.

Spencer K. A. 1985. East African Agromyzidae (Diptera): further descriptions, revisionary notes and new records. J. Nat. Hist. 19: 969-1027.

Spencer K. A., Steyskal G. C. 1986. Manual of the Agromyzidae (Diptera) of the United States. Handbook 638. U.S. Department of Agriculture, Washington, DC.

Spencer K. A. 1990. Host specialisation in the world Agromyzidae (Diptera). Kluwer Academic Publishers, Dordrecht, The Netherlands.

Sugimoto T., Kawado K., Tadera K. 1988. Responses to the host mine by two parasitic wasps, Dapsilarthra rufiventris (Hymenoptera: Braconidae) and Chrysocharis pentheus (Hymenoptera: Eulophidae). J. Ethol. 6: 55-58.

Thang V. T. 1999. Surveys of leafminers (Liriomyza) and their parasitoids on vegetables in Vietnam 1998, pp 42-53. In Lim G. S., Soetikno S. S., Loke W. H. (eds.), Proceedings of a Workshop on Leafminers of Vegetables in South East Asia, 2-5 February 1999, Tanah Rata, Malaysia. CAB International Southeast Asia Regional Centre, Serdang, Malaysia.

van Driesche R. G., Taub G. 1983. Field evaluation of the impact of parasites on Phyllonorycter leafminers infesting apple in Massachusetts, U.S.A. Prot. Ecol. 5: 305-317.

Wei J.-N., Zuh J., Kang L.. 2006. Volatiles released from bean plants in response to agromyzid flies. Planta 224: 279-287.

Zhao Y. X., Kang L. 2002. The role of plant odours in the leafminer Liriomyza sativae (Diptera: Agromyzidae) and its parasitoid Diglyphus isaea (Hymenoptera: Eulophidae): orientation towards the host habitat. Eur. J. Entomol. 99: 445-450 